\title{
Rectal and colonic mucosal biopsy findings and faeces, sigmoidoscopy, and histopathological correlation in amoebiasis and other colitis ${ }^{1}$
}

\author{
N. MADANAGOPALAN, S. P. VEDACHALAM, R. SUBRAMANIAM, AND \\ R. G. MURUGESAN
}

From Madras Medical College and the Government General Hospital, Madras, India

It is rather surprising that despite the ease of obtaining a rectal biopsy and considerable experience of this technique which exist in the routine diagnosis of neoplastic lesions in the rectum, it is only over the last two decades that its application in inflammatory lesions of the colon and rectum has become popular.

As amoebiasis is very common in Madras, it had become a necessity to exclude it in all cases presenting with bowel disturbances and vague dyspeptic states, particularly if associated with hepatomegaly. Further, amoebiasis coexists or complicates other colonic or rectal lesions. The various methods available for establishing the diagnosis of amoebiasis have been the demonstration of $(a)$ the vegetative form of Entamoeba histolytica in the faeces, or rectal swab, or scrapings from ulcer or aspirated material from suspected sites or in tissues obtained at biopsy; (b) the cystic form of $E$. histolytica in faeces; $(c)$ the demonstration of rectal and colonic lesions on sigmoidoscopy; $(d)$ the complementfixation test; (e) fluorescent microscopy, and, $(f)$ the clinical impression and response to antiamoebic treatment.

Juniper (1962) observed that 18 out of 22 patients in whom ulcers were seen sigmoidoscopically showed diagnostic trophozoites in mucosal biopsy specimens. Doxiades and Yiotsas (1965) observed amoeba by light microscopy not only in the walls of ulcers as noted by others, but also in $37 \%$ of rectal and colonic mucosal biopsy material from regions which were apparently normal at sigmoidoscopy. If this could be corroborated we feel that it will be a very useful procecure in establishing a definite diagnosis in such cases of colitis, non-ulcer dyspepsias, hepatomegaly, and pleuro-pulmonary lesions which are sometimes treated as amoebic in aetiology purely on clinical impressions.

'Paper read at the 7th Annual Conference of the Indian Society of Gastroenterology held at Bombay during September 1966.
MATERIAL

We are presenting a preliminary report of the colonic mucosal biopsy in 115 patients, in whom a routine faeces examination, proctosigmoidoscopy, and rectal and colonic mucosal biopsy using a long alligator forceps with sharp cupped blades were done. This procedure was carried out without any prior preparation of the bowel.

The material obtained on rectal swabs and by sigmoidoscopy was examined under the microscope as a routine and the findings on sigmoidoscopy were recorded. The biopsy specimens were fixed immediately in formalin and then embedded in paraffin. Subsequently paraffin sections were all stained with haematoxylin and eosin. In those where the presence of E. histolytica was suspected on histopathological examination, further sections were taken and stained with iron haematoxylin.

Included in this study are 36 cases of acute amoebic dysentery, 12 of chronic intestinal amoebiasis, nine of hepatic amoebiasis, four of pleuro-pulmonary amoebiasis, one of cutaneous amoebiasis, six of bacillary dysentery, nine of ulcerative colitis, 12 of irritable colon, 16 of tuberculous abdomen, and 10 miscellaneous cases.

\section{RESULTS}

ACUTE AMOEBIC DYSENTERY (36) All these patients presented with acute dysenteric symptoms and 34 of 36 had the vegetative form of $E$. histolytica in faeces and the cystic form was found in one. Sigmoidoscopy revealed typical ulceration (multiple or solitary or in groups) or haemorrhagic colitis or thick whitish membranous lesions in 33 . Only in three, were no lesions apparent on sigmoidoscopy.

The histopathological examination showed ulcers with exudative lesions in 17 , crypt abscesses without actual ulceration in two, and focal lymphocytic collections in nine. However, the significance of a focal lymphocytic collection is not apparent, as will be mentioned later. The haemotoxylin and eosin stain revealed suspicion of amoeba in 10 but could be confirmed with the iron-haematoxylin stain in 
TABLE I

FAECES, SIGMOIDOSCOPY, AND HISTOLOGICAL CORRELATION IN AMOEBIASIS

\begin{tabular}{llll}
$\begin{array}{l}\text { Total } \\
\text { No. of } \\
\text { Cases }\end{array}$ & $\begin{array}{l}\text { Faeces } \\
\text { E. histolytica } \\
\text { (veg.) }\end{array}$ & Cyst & $\begin{array}{l}\text { Positive } \\
\text { Sigmoidoscopy } \\
\text { Findings }\end{array}$ \\
\hline
\end{tabular}

Histopathology

33

Acute amoebic dysentery

Chronic intestinal amoebiasis

Hepatic amoebiasis

Pleuro-pulmonary amoebiasis

Cutaneous

Total

only five. One such case, which was not confirmed with iron-haematoxylin staining, presented with a dysenteric illness of 10 months' duration. The routine examination of the faeces and sigmoidoscopy were not contributory but the patient made a dramatic recovery with anti-amoebic treatment. Hence, that case is also included in this group. Sigmoidoscopy detected lesions in $91.7 \%$ of proven cases of acute amoebic dysentery while mucosal biopsy was confirmative in only $13.9 \%$ of cases.

The faeces, sigmoidoscopic, and histopathological correlation observed in cases of amoebiasis studied are presented in Table $\mathrm{I}$.

Figure 1 shows the amoeba in tissue demonstrated by iron-haemotoxylin staining.

Figure 2 shows a microsçopic crypt abscess in a patient who presented with acute dysenteric symptoms, plenty of $E$. histolytica in faeces, but the mucosa was apparently normal on sigmoidoscopy. It is possible amoebae were travelling down from ulcers higher up. However, it shows that crypt abscess could well be an early lesion in amoebiasis as in any diffuse inflammatory lesion of the gut.

CHRONIC INTESTINAL AMOEBIASIS (12) These patients presented with recurrent attacks of diarrhoea or dysentery. The cystic form of $E$. histolytica was demonstrated in nine and sigmoidoscopy revealed typical amoebic ulcers in the remaining three. Besides these three, one of the patients who had the cyst in faeces had an ulcer demonstrated at sigmoidoscopy while three had a granular mucosa not unlike that observed in ulcerative colitis in an inactive form. Mucosal biopsy was done in all cases; an ulcer with an exudative lesion was observed in one, a crypt abscess in one, and focal lymphocytic collections in three. In none was $E$. histolytica demonstrated in the tissues.
Figure 3 shows a crypt abscess working up, lifting the mucosa, something like that which is observed in skin about to ulcerate, in a patient who had vague dyspepsia. The examination of faeces and sigmoidoscopy were normal, and he improved markedly after anti-amoebic therapy.

HEPATIC AMOEBIASIS (9) All these cases had tender hepatomegaly, a previous history of dysentery, and some were alcoholic. Indeed three cases were proven to be of liver abscess, anchovy sauce material being obtained on needling. Examination of faeces did not reveal $E$. histolytica in any of these nine cases. While sigmoidoscopy revealed lesions in the colon in three (ulcerations in two and granularity in one) $E$. histolytica could not be demonstrated in the tissue obtained at biopsy. The significant abnormalities observed histopathologically were ulceration in one, crypt abscess in one, and a focal lymphocytic collection in one.

Figure 4 demonstrates the microscopic abscess with focal lymphocytic collection in a person who was proved to have a left lobe liver abscess.

PLEURO-PULMONARY AMOEBIASIS (4) Included in this group were cases with a previous history of amoebiasis, hepatomegaly, and shadowing in the right lower lobe and all of them responded to antiamoebic therapy. Examination of faeces was noncontributory in all and sigmoidoscopy revealed ulcer in two and microscopic ulcer was demonstrated on histopathological examination in one. In none was $E$. histolytica seen in tissues.

CUTANEOUS AMOEBIASIS This case presented with a sloughing ulcer in the scrotum (Fig. 5) and dysentery. Faeces and the exudate from the ulcer in the scrotum were teeming with $E$. histolytica. Multiple 

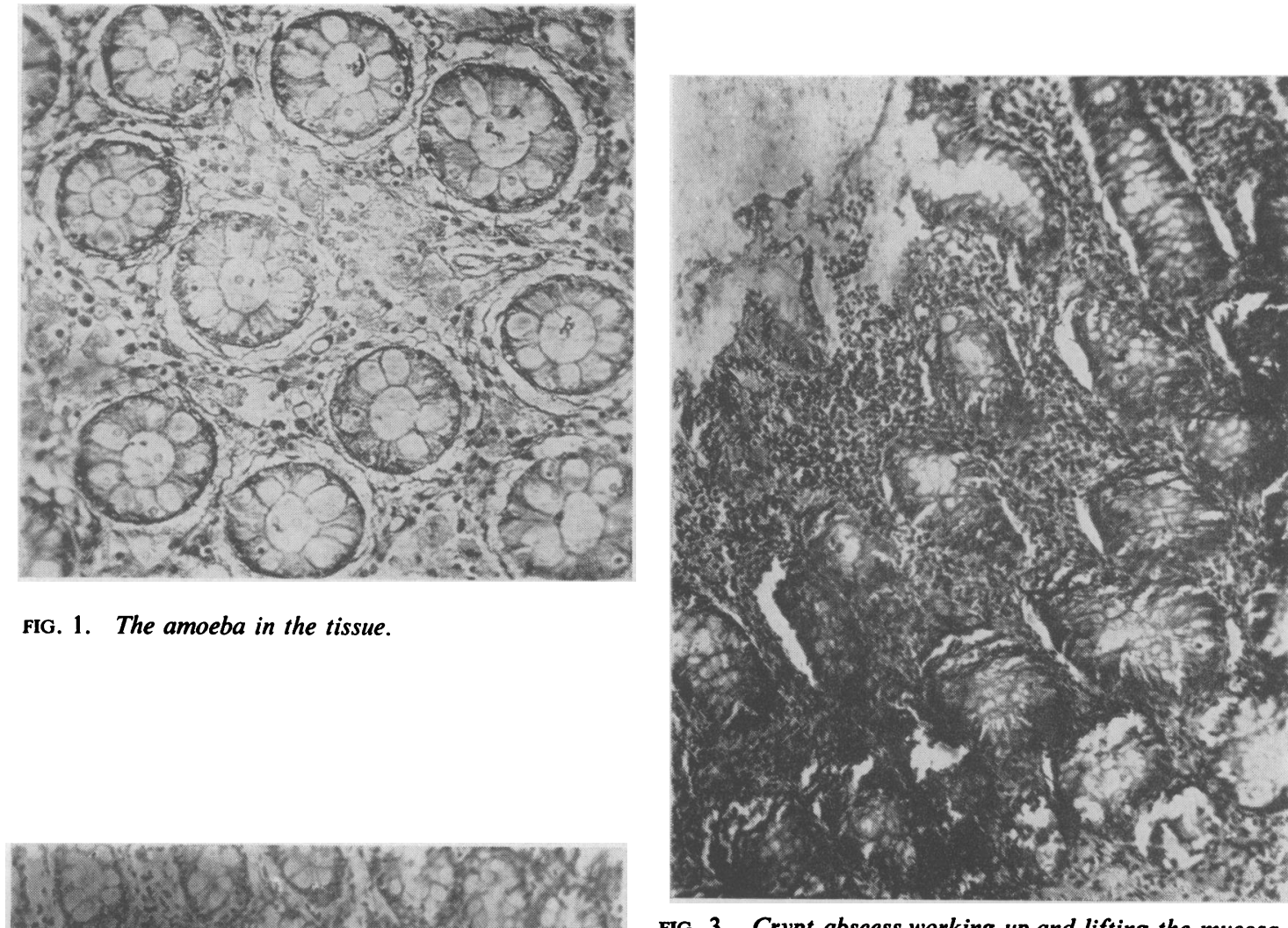

FIG. 1. The amoeba in the tissue.

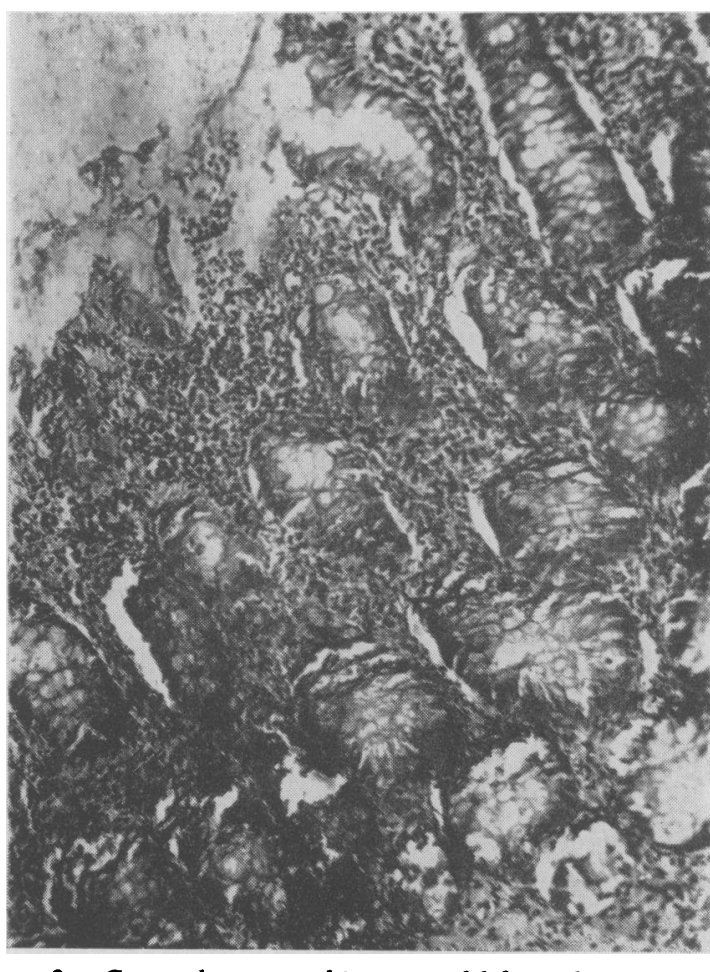

FIG. 3. Crypt abscess working up and lifting the mucosa.

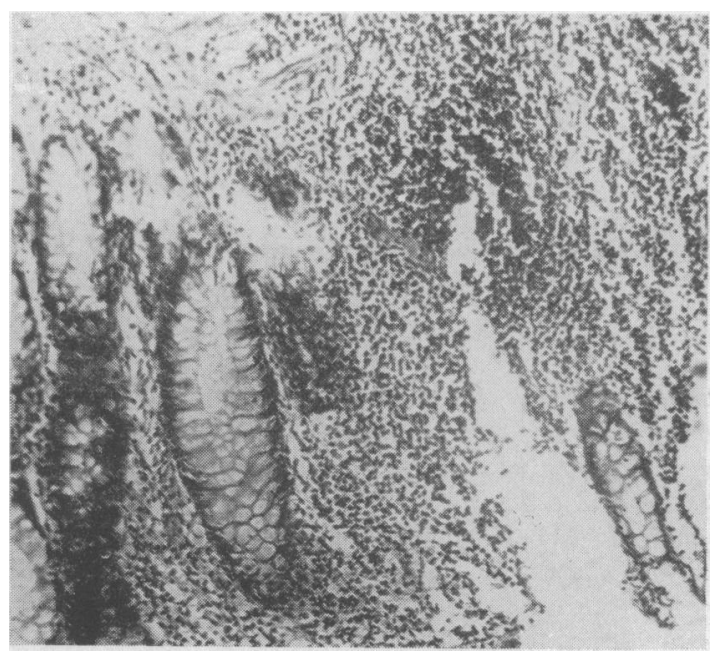

FIG. 4. Lymphocytic collection and crypt abscess.

FIG. 2. The crypt abscess. 


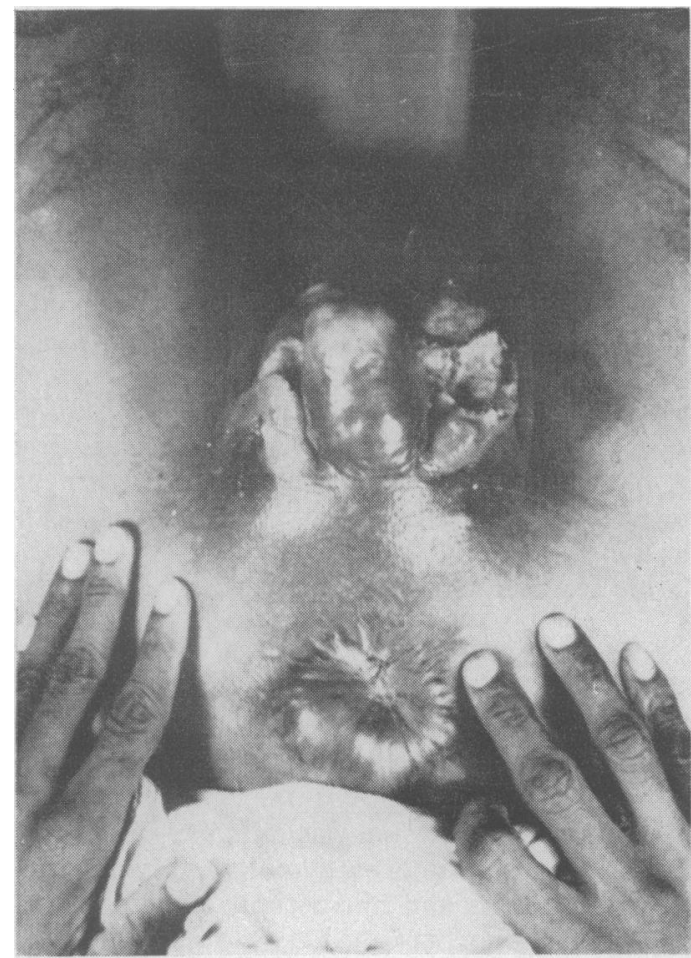

FIG. 5. Amoebic ulceration of scrotum.

ulcers were seen at sigmoidoscopy. However, no $E$. histolytica were seen in tissue obtained by rectal and colonic mucosal biopsy which showed only superficial ulcerations.

BACILLARY DYSENTERY (6) All these patients presented with a sudden onset of dysentery with a high fever and constitutional symptoms, their faeces were negative for $E$. histolytica, and they responded to sulphaguanidine and/or chloramphenicol. Extensive ulcerations were observed sigmoidoscopically in three, haemorrhagic colitis in two, and a membranous slough in one. While all showed ulceration, severe exudative lesions, consisting predominantly of neutrophils, were observed only in three. The sigmoidoscopic and histopathological appearances observed in this and other conditions described subsequently are summarized in Table II.

? ULCERATIVE COLITIS (9) These patients presented with a long history of recurrent episodes of diarrhoea with blood and mucus intimately mixed, in whom no other cause could be detected on detailed investigation and anti-amoebic therapy failed to make any impression. Haemorrhagic proctocolitis was observed at sigmoidoscopy in three, and in the other six there was granularity of the mucosa, contact bleeding, and loss of vascular pattern. The mucosal biopsy revealed ulcerated and haemorrhagic mucosa in six, congested mucosa in two, and was almost normal in one. No amoebae were seen in the tissues.

TUBERCULOUS ABDOMEN (16) These were all patients with diarrhoea with mucus and with or without blood who had either proven pulmonary tuberculosis or a violent Mantoux reaction, in whom routine faeces examination did not reveal $E$. histolytica. Superficial ulcerations were noted in four, granularity in two, and oedema of the mucosa with loss of vascular pattern in two not unlike that observed in ulcerative colitis in inactive form. The histopathological examination revealed mucosal ulceration in one, an exudative lesion in one, and focal lymphocytic collections in five. None showed any caseation or giant cell.

IRRITABLE COLON (12) These were patients who were worried by the presence of mucus in the faeces, occasional tenesmus, and vague abdominal discomfort in whom all routine investigations were non-contributory. The faeces did not reveal any pathogen microscopically and sigmoidoscopy was

TABLE II

FINDINGS IN OTHER CONDITIONS STUDIED

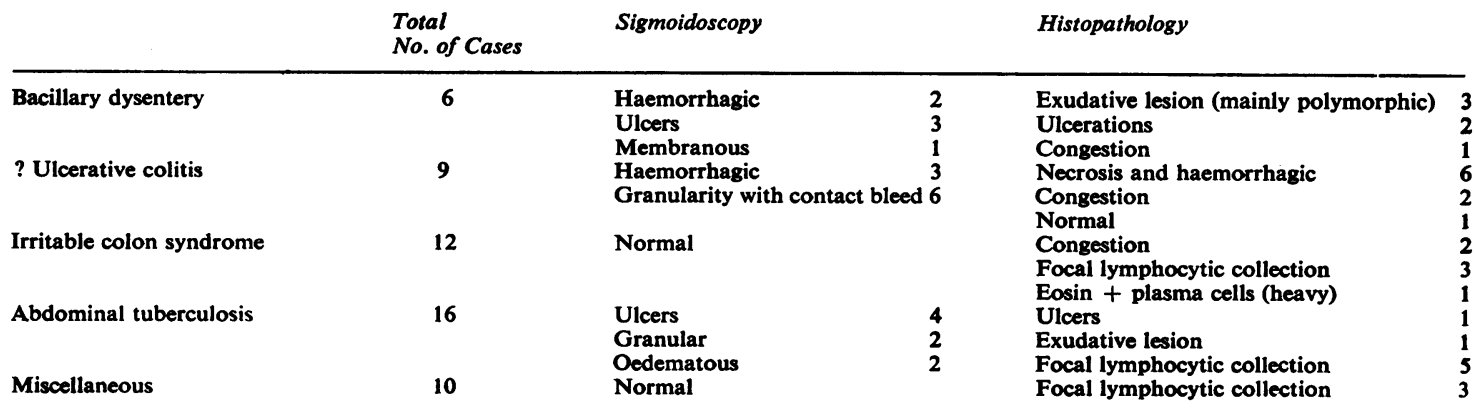


normal in all. The histopathological examination revealed congestion in two, focal lymphocytic collections in three, and heavy eosinophilic and plasma cell infiltration in one.

MisCellaNEOUS (10) The cases included in this group are unexplained hepatomegaly two, Hodgkin's disease one, cancer of liver one, multiple myeloma one, carcinoma of the anal canal one, gallstone with obstructive jaundice one, Bowen's disease one, diabetes mellitus one, and duodenal ulcer one.

Sigmoidoscopy was normal in all of them and the only abnormality in the histopathological examination of the mucosa was the presence of focal lymphocytic collections in three. Two of these had ascariasis and one was a case of multiple myeloma.

\section{DISCUSSION}

In this study, we have observed that sigmoidoscopy has detected lesions in $91.7 \%$ of proven cases of acute amoebic dysentery while in only $13.9 \%$ could the amoeba be demonstrated in rectal and colonic mucosal biopsy. Colonic mucosal biopsy did not reveal $E$. histolytica in any case of chronic intestinal amoebiasis or hepatic or pleuropulmonary or cutaneous amoebiasis in this series. This is at variance with the finding of Doxiades and Yiotsas (1965) who observed E. histolytica in tissues obtained by rectal and colonic mucosal biopsy in 238 of 1,054 cases $(22.6 \%)$ with no evidence of active colitis sigmoidoscopically but were suspected as having amoebiasis. Indeed, the majority did not show $E$. histolytica in faeces. As the procedure of colonic mucosal biopsy does carry with it a potential risk and need for special studies, it is felt that useful information can be obtained by careful and repeated examination of faeces and/or rectal swab for $E$. histolytica coupled with sigmoidoscopy rather than to depend on colonic mucosal biopsy too much for a positive result.

In places where radical surgery on the colon and rectum is undertaken on a presumptive diagnosis of ulcerative colitis even an occasional demonstration of $E$. histolytica in tissue might mean saving the patient from the disadvantages of ileostomy. But, in Madras, where the necessity for such procedures in dysenteric illnesses is almost nil too much stress need not be laid on colonic mucosal biopsy, apart from excluding other serious illnesses.

Morson (1965) has observed that crypt abscess is not specific to ulcerative colitis and that it could occur in any diffuse inflammation of the gut. Crypt abscesses are also observed in amoebiasis as shown in our cases. Morson has observed focal lymphadenoid hyperplasia in Crohn's disease and, in his opinion, the fissuring in Crohn's disease begins in the mucosa at the site of lymphadenoid aggregations. The detection of focal lymphocytic collections observed at mucosal biopsy in amoebiasis $(21 \%)$, abdominal tuberculosis $(31.3 \%)$, and the irritable colon syndrome $(25 \%)$ in our series makes us suspect its possible role in the pathogenesis of ulceration in colonic mucosa, whatever be the aetiology. However, $30 \%$ in a miscellaneous group also had focal lymphocytic collections. Whether this finding is only a chance observation or a significant one, a follow up of these cases and further studies on a larger scale might provide an answer.

\section{SUMMARY}

We have observed that examination of faeces and sigmoidoscopy yield more positive results than rectal and colonic mucosal biopsy in amoebiasis. A thick, white, membranous lesion was a finding observed in a few cases of acute amoebic dysentery. It is suggested that crypt abscess and microscopic ulcerations can also occur in amoebiasis.

Non-specific ulceration, granularity, and oedema with loss of vascular pattern, such as those recorded in ulcerative colitis in inactive form, were also observed in some cases of proven abdominal tuberculosis. The presence of focal lymphocytic collec ions in colonic and rectal mucosa and its possible role in the genesis of ulceration is discussed.

Our grateful thanks are due to all the medical officers who referred their cases to us in this study. Special thanks are due to Professor P. N. Rangiah, Director, Institute of Venereology, General Hospital, Madras, for not only referring but also permitting us to publish the clinical photograph of the case of cutaneous amoebiasis.

We take this opportunity to thank the Dean, General Hospital, and the Director of Medical Education, Madras, for permitting us to present and publish this paper.

\section{REFERENCES}

Doxiades, T., and Yiotsas, Z. (1965). The importance of rectal biopsy in the diagnosis of amebiasis. Amer. J. Gastroent., 43, 229-234. Juniper, K., Jr. (1962). Acute amebic colitis. Amer. J. Med., 33, 337-386.

Morson, B. C. (1965). Crohn's disease of the small intestines (and discussion). In The Small Intestine. Symposium of the Sth Congress of the International Acaden $y$ (f Pathclcgy. Fdited by A. C. Thackray and F. A. Jones. pp. 98-109. Blackwell, Oxford. 